\title{
Associations of oxidative stress and inflammation and their role in the regulation of membrane fluidity of red blood cells in hypertensive and normotensive men: An electron spin resonance investigation
}

\author{
Kazushi Tsuda ${ }^{1,2}$ \\ ${ }^{1}$ Cardiovascular and Metabolic Research Center, Kansai University of Health Sciences, Osaka, Japan \\ ${ }^{2}$ Division of Cardiology, Department of Medicine, Wakayama Medical University, Wakayama, Japan \\ Email: tsudak@mail.wakayama-med.ac.jp
}

Received 19 August 2012; revised 27 September 2012; accepted 13 October 2012

\begin{abstract}
There is evidence showing that increased levels of oxidative stress and C-reactive protein (CRP) might be associated with obesity, hypertension, atherosclerosis and other cardiovascular diseases. This study was undertaken to investigate possible relationships among plasma 8-iso-prostaglandin F2 $\alpha$ (8-iso-PG F2 $\alpha$ : an index of oxidative stress), high-sensitivity (hs)CRP and membrane fluidity (a reciprocal value of microviscosity) in hypertensive and normotensive men using an electron spin resonance (ESR)-method. The order parameter $(\mathrm{S})$ for the spin-label agents (5nitroxide stearate) of red blood cell (RBC) membranes in the ESR spectra was significantly higher in hypertensive men than in normotensive men, indicating that membrane fluidity was decreased in hypertensive men. Both plasma 8-iso-PG F2 $\alpha$ and hsCRP levels were significantly increased in hypertensive men compared with normotensive men. In addition, plasma plasma 8-iso-PG F2 $\alpha$ levels were correlated with plasma hs-CRP levels. In contrast, plasma nitric oxide (NO)-metabolites were lower in hypertensive men than in normotensive men, and inversely correlated with plasma 8-iso-PG F2 $\alpha$ and hs-CRP. The order parameter(S) of RBCs was correlated with plasma 8-iso-PG F2 $\alpha$ and plasma hs-CRP, and inversely correlated with plasma NO-metabolites, suggesting that reduced membrane fluidity of RBCs might be associated with increased oxidative stress, inflammation and endothelial dysfunction. Multivariate regression analysis also showed that, after adjusting for general risk factors, both plasma 8iso-PG F2 $\alpha$ and hs-CRP were significant determinants of membrane fluidity of RBCs. The ESR suggests that associations of oxidative stress and inflammation might have a close correlation with impaired
\end{abstract}

rheologic behavior of RBCs and microcirculatory dysfunction in hypertensive men.

Keywords: Oxidative Stress; 8-Iso-Prostaglandin F2 $\alpha$; High-Sensitivity C-Reactive Protein; Nitric Oxide;

Membrane Fluidity; Electron Spin Resonance;

Hypertension

\section{INTRODUCTION}

Evidence indicates that oxidative stress might actively participate in the pathophysiology of obesity, hypertension and atherosclerosis, and might be associated with increased risk of cardiovascular diseases, vascular dysfunction, and the metabolic syndrome [1-4]. Recently, it has been shown that plasma 8-iso-prostaglandin F2 $\alpha$ (8-iso-PG F2 $\alpha$ ) may be a reliable index of oxidative stress in humans [5-8]. It was demonstrated that plasma concentration of 8-iso-PG F2 $\alpha$ was significantly increased in subjects with essential hypertension compared with normotensive subjects $[5,6]$. It was also shown that plasma 8-iso-PG F2 $\alpha$ levels were elevated in patients with coronary artery disease, particulary in those with hypertension $[7,8]$.

On the other hand, it is also well recognized that inflammation might be involved in the development and progression of cardiovascular disease processes [9]. Recent studies have shown that high-sensitivity C-reactive protein (hs-CRP), an acute-phase of inflammatory marker, might be associated with increased risk of hypertension and other circulatory disorders [10-14].

Abnormalities in physical properties of the cell membranes may underlie the defects that are strongly linked to hypertension, stroke, and other cardiovascular disease conditions $[15,16]$. An electron spin resonance (ESR) and spin-labeling method has been developed to evaluate membrane fluidity (a reciprocal value of membrane mi- 
croviscosity) and perturbations of the membrane function by external agents $[15,16]$. Using the ESR method, we have been performing a series of experiments regarding the membrane fluidity in subjects with essential hypertension [16-20]. The results suggest that membrane fluidity of red blood cells (RBCs) was significantly lower in hypertensive subjects than in normotensive subjects, indicating that the cell membranes were stiffer and less fluid in essential hypertension [16-20]. Because the deformability of RBCs may be highly dependent on membrane fluidity [15], the reduction in membrane fluidity of RBCs could cause a disturbance in the blood rheologic behavior and in the microcirculation, which might contribute to the pathophysiology of hypertension and other circulatory disorders $[15,16]$. In the studies presented previously, we showed that plasma 8-iso-PG F2 $\alpha$ and hs-CRP levels might be associated with reduced membrane fluidity of RBCs, respectively [21,22], although associations of oxidative stress and inflammation and their role in the regulation of membrane fluidity in hypertension remain to be solved. In the present study, therefore, we investigated the relationships among plasma 8 -iso-PG F2 $\alpha$, hs-CRP and membrane fluidity of RBCs in hypertensive and normotensive men using the ESR method.

\section{SUBJECTS AND METHODS}

\subsection{Subjects}

A total of 30 men with untreated essential hypertension (systolic blood pressure more than $140 \mathrm{mmHg}$ and/or diastolic blood pressure more than $90 \mathrm{mmHg}$ ) were studied and compared with 23 age-matched normotensive men. The characteristics and laboratory findings in both groups were shown in Table 1. Subjects who had a history of other diseases such as haematologic or hepatic disorders were excluded. All men were non-smokers. They had similar life styles and dietary habits, and were instructed to avoid any changes in dietary habits before the study. The study was approved by a local research committee of Kansai University of Health Sciences. Informed consent was obtained from all participants after they were informed about the nature and objective of the study.

\subsection{Electron Spin Resonance (ESR) Measurements of Red Blood Cells (RBCs)}

Blood sampling was performed by venipuncture after a 30 minutes of bed rest while fasting. The procedures of RBC preparation and ESR measurements were shown previously [17-20]. The washed RBCs were suspended in the isotonic buffer $(140 \mathrm{mmol} / \mathrm{L} \mathrm{NaCl}$ and $20 \mathrm{mmol} / \mathrm{L}$ Tris- $\mathrm{HCl}$ buffer, $\mathrm{pH} 7.4$ ) at a heamtocrit of $50 \%$. The RBC suspension was incubated with the solution con-
Table 1. Clinical characteristics and laboratory findings of hypertensive (HT) and normotensive (NT) men.

\begin{tabular}{lcc}
\hline & NT & HT \\
\hline Number of subjects & 23 & 30 \\
Age (y.o.) & $64 \pm 2$ & $62 \pm 2$ \\
Body mass index (kg/m²) & $23.4 \pm 0.7$ & $24.3 \pm 0.6$ \\
Systolic blood pressure (mm Hg) & $124 \pm 2$ & $145 \pm 1^{*}$ \\
Diastolic blood pressure (mm Hg) & $69 \pm 2$ & $84 \pm 1^{*}$ \\
Heart rate (beats/min) & $76 \pm 1$ & $73 \pm 1$ \\
Erythrocyte counts $\left(10^{4}\right.$ cells/ $\left.\mu \mathrm{L}\right)$ & $455 \pm 10$ & $472 \pm 8$ \\
Hemoglobin (g/dL) & $14.2 \pm 0.3$ & $14.1 \pm 0.2$ \\
Hematocrit (\%) & $43.5 \pm 0.9$ & $41.9 \pm 1.3$ \\
Leucocyte counts $\left(10^{3}\right.$ cells/ $\left.\mu \mathrm{L}\right)$ & $5.6 \pm 0.3$ & $5.5 \pm 0.2$ \\
Platelets (10 ${ }^{4}$ cells/ $\left.\mu \mathrm{L}\right)$ & $20 \pm 1$ & $22 \pm 1$ \\
Total cholesterol $(\mathrm{mg} / \mathrm{dL})$ & $207 \pm 6$ & $210 \pm 6$ \\
High density lipoprotein cholesterol & $53 \pm 2$ & $54 \pm 3$ \\
(mg/dL) & & \\
Low density lipoprotein cholesterol & $128 \pm 5$ & $125 \pm 6$ \\
(mg/dL) & $113 \pm 8$ & $165 \pm 31$ \\
Triglycerides (mg/dL) & $140.7 \pm 0.4$ & $140.2 \pm 0.3$ \\
Serum sodium (mmol/L) & $4.0 \pm 0.1$ & $4.0 \pm 0.1$ \\
Serum potassium (mmol/L) & $0.8 \pm 0.1$ & $0.9 \pm 0.1$ \\
Serum creatinine (mg/dL) & $119 \pm 8$ & $125 \pm 8$ \\
Fasting plasma glucose (mg/dL) & & \\
\hline
\end{tabular}

Values are means \pm SEM. ${ }^{*} \mathrm{P}<0.01$ between HT and NT.

taining fatty acid spin label agent (5-nitroxide stearate 5 $\times 10^{-5} \mathrm{~mol} / \mathrm{L}$, Aldrich Co. Ltd., Milwaukee, Wisconsin, USA) for 2 hours at $37^{\circ} \mathrm{C}$ with gentle shaking [17-20]. After incubation, the ESR measurements were immediately performed using an ESR spectrometer (Model Jeol JES-FE2XG, Nihon Denshi, Tokyo, Japan) with a microwave unit (Model Jeol ES-SCXA, Nihon Denshi). The microwave power was $5 \mathrm{~mW}$ and the modulation frequency was $100 \mathrm{kHz}$ with a modulation amplitude of 0.2 $\mathrm{mT}$ (tesla) [17-20]. For indices of membrane fluidity, we evaluated the values of outer and inner hyperfine splitting (2 $\mathrm{T}^{\prime} / /$ and $2 \mathrm{~T}^{\prime} \perp$ in $\mathrm{T}$, respectively) in the ESR spectra and calculated the order parameter (S) (Figure 1) [17-20]. The greater the value of the order parameter (S) was, the lower the membrane fluidity of RBCs was [1720].

\subsection{Analaysis of Plasma 8-Iso-Prostaglandin F2 $\alpha$ and Plasma Nitric Oxide (NO)-Metabolites (Nitrite and Nitrate)}

Plasma 8-iso-PG F2 $\alpha$ levels were determined by using an enzyme immunoassay (Cayman Chemicals Co., Ann Arbor, Michigan, USA) [6,7]. The plasma levels of NOmetabolites (nitrite and nitrate) were measured with an automated NO detector/high-performance liquid chromatography system (ENO 10, Eicom Co., Tokyo, Japan), as 


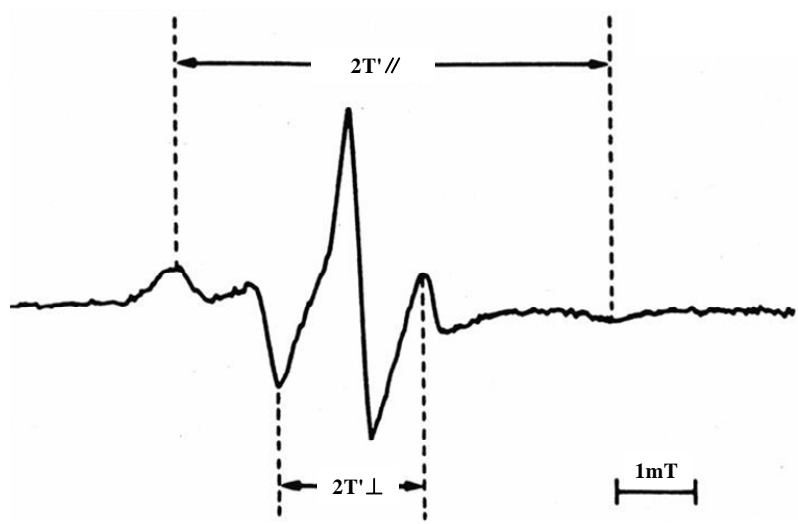

Figure 1. Representative electron spin resonance spectrum of red blood cells (RBCs) for the fatty acid spin-label agent (5nitroxide stearate). $2 \mathrm{~T}^{\prime} / /$ : outer hyperfine splitting, $2 \mathrm{~T}^{\prime} \perp$ : inner hyperfine splitting, T: tesla; We calculated the order parameter (S) from $2 \mathrm{~T}^{\prime} / /$ and $2 \mathrm{~T}^{\prime} \perp$ values according to the formula [17-20]; $S=\left(a_{n} / a_{n}^{\prime}\right)\left(T^{\prime} / /-T^{\prime} \perp\right) /(T z z-T x x) ; a_{n} / a_{n}^{\prime}$ : isotropic coupling constant, Tzz and Txx: hyperfine constants; The greater the value of the order parameter $(\mathrm{S})$ was, the lower the membrane fluidity of red blood cells (RBCs) was [17-20].

previously described (20). Briefly, nitrite and nitrate in plasma were separated by a reverse-phase separation column, and the nitrate was reduced to nitrite in a reduction column. Nitrite was mixed with Griess reagents (sulfanilamide and naphthalene-ethylene diamine dihydrochloride), and the absorbance at $540 \mathrm{~nm}$ was measured by a flow-through spectrometer.

\subsection{Statistical Analysis}

Values are expressed as mean \pm SEM. The differences between hypertensive and normotensive men were analyzed using an unpaired Student's t-test. Linear regression analysis was performed to assess the relationships among membrane fluidity (order parameter; S) of RBCs and plasma 8-iso-PG F2 $\alpha$, hs-CRP, and NO-metabolite levels. Multivariate regression analysis with membrane fluidity (order parameter; S) of RBCs as a dependent variable, and general risk factors (age, body mass index; BMI, total cholesterol, fasting blood glucose and systolic blood pressure), plasma 8-iso-PG F2 $\alpha$, and plasma hs$\mathrm{CRP}$ as independent variables was also performed. A P value less than 0.05 was accepted as the level of significance.

\section{RSULTS}

\subsection{Membrane Fluidity of RBCs in Hypertensive and Normotensive Men}

The order parameter (S) for the spin-label agents (5-nitroxide stearate) of RBC membranes in the ESR spectra was significantly higher in hypertensive men (HT: 0.727 $\pm 0.002, \mathrm{n}=30$ ) than in normotensive men (NT: $0.717 \pm$
$0.002, \mathrm{n}=23, \mathrm{P}<0.001$ ), indicating that membrane fluidity was decreased in hypertensive men.

\subsection{Plasma 8-Iso-PG F2 $\alpha$, Plasma High-Sensitivity C-Reactive Protein, and Plasma NO-Metabolite Levels in Hypertensive and Normotensive Men}

The plasma 8-iso-PG F2 $\alpha$ levels were significantly higher in hypertensive men than in normotensive men (HT: $2.89 \pm 0.29 \mathrm{nmol} / \mathrm{L}, \mathrm{n}=30$, NT: $1.78 \pm 0.21 \mathrm{nmol} / \mathrm{L}, \mathrm{n}=$ 23, $\mathrm{P}<0.01$ ). Plasma hs-CRP levels were also elevated hypertensive men compared with normotensive men (HT $0.147 \pm 0.019 \mathrm{mg} / \mathrm{dL}, \mathrm{n}=30$, NT $0.080 \pm 0.009 \mathrm{mg} / \mathrm{dL}$, $\mathrm{n}=23, \mathrm{P}<0.01)$. In contrast, the plasma NO-metabolites were lower in hypertensive men than in normotensive men (HT: $38.4 \pm 2.8 \mu \mathrm{mol} / \mathrm{L}, \mathrm{n}=30$, NT: $52.5 \pm 4.4$ $\mu \mathrm{mol} / \mathrm{L}, \mathrm{n}=23, \mathrm{P}<0.01)$.

In the overall analysis of hypertensive and normotensive men, plasma 8 -iso-PG F2 $\alpha$ levels were correlated with plasma hs-CRP levels $(\mathrm{r}=0.395, \mathrm{n}=53, \mathrm{P}<0.01)$ (Figure 2).

In addition, plasma NO-metabolites were inversely correlatd with plasma 8-iso-PG F2 $\alpha(\mathrm{r}=-0.290, \mathrm{n}=53$, $\mathrm{P}<0.05)$ (Figure 3) and plasma hs-CRP $(\mathrm{r}=-0.277, \mathrm{n}=$ $53, \mathrm{P}<0.05)$ (Figure 4).

\subsection{Relationship among Membrane Fluidity of RBCs, Plasma 8-Iso-PG F2 $\alpha$, Plasma High-Sensitivity C-Reactive Protein, and NO-Metabolite Levels in Hypertensive and Normotensive Men}

The order parameter (S) of RBC membranes in the ESR spectra was correlated with plasma 8-iso-PG F2 $\alpha(\mathrm{r}=$ $0.434, \mathrm{n}=53, \mathrm{P}<0.01)$ (Figure 5) and plasma hs-CRP $(\mathrm{r}$ $=0.378, \mathrm{n}=53, \mathrm{P}<0.01)$ (Figure 6), and inversely correlated with plasma NO-metabolites $(\mathrm{r}=-0.322, \mathrm{n}=53$, $\mathrm{P}<0.05$ ) (Figure 7). These findings suggest that reduced membrane fluidity of RBCs might be associated with increased oxidative stress, inflammation and endothelial dysfunction.

Multivariate regression analysis also showed that, after adjustment for general risk factors, both plasma 8-iso-PG F2 $\alpha$ and hs-CRP levels were significant determinants of membrane fluidity (order parameter; S) of RBCs (Table 2).

\section{DISCUSSION}

Evidence indicates that both oxidative stress and hs-CRP might be strongly related to the pathophysiology of hypertension, atherosclerosis, and other cardiovascular diseases $[10,11]$. The present study was performed to evaluate the possible relationships among plasma 8-iso-PG F $2 \alpha$ (an index of oxidative stress), hs-CRP and membrane fluidity of RBCs in hypertensive and normotensive 


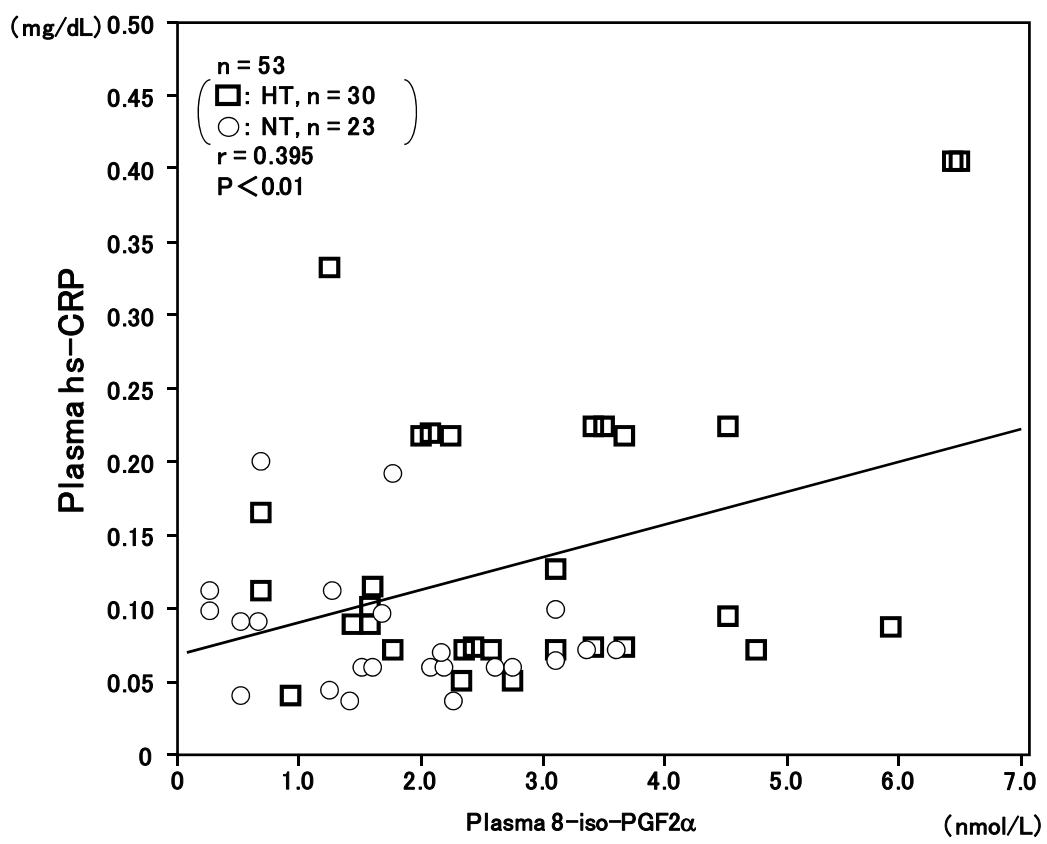

Figure 2. Correlation between plasma 8-iso-PG F2 $\alpha$ and plasma high-sensitivity $\mathrm{C}$-reactive protein (hs-CRP) levels in hypertensive and normotensive men.

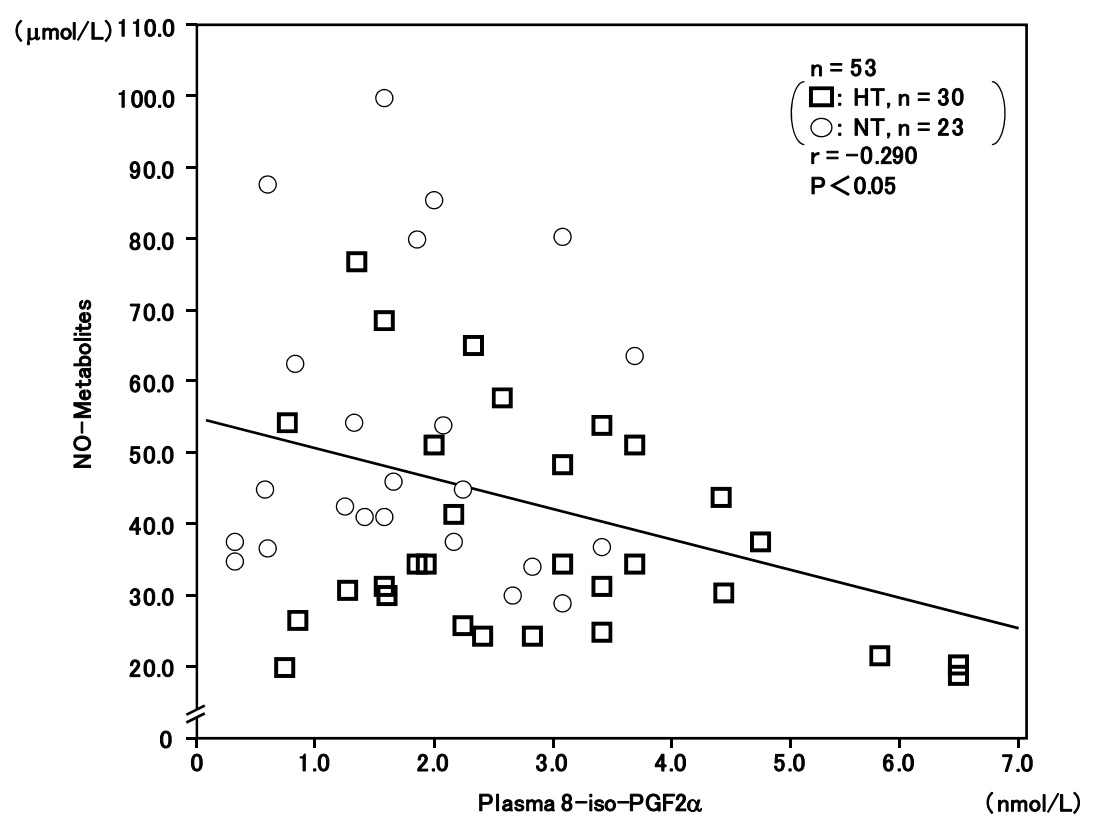

Figure 3. Inverse correlation between plasma 8-iso-PG F2 $\alpha$ and plasma NO-metabolite levels in hypertensive and normotensive men.

men using the ESR method. The results showed that the order parameter (S) of RBC membranes in the ESR spectra was significantly higher in hypertensive men than in normotensive men, indicating that membrane fluidity of RBCs was decreased in hypertension. The result might be consistent with our previous findings showing that the cell membranes were stiffer and less fluid in hypertension $[16-20]$.
In the present study, we showed that plasma 8-iso-PG F2 $\alpha$ and hs-CRP levels were significantly higher in hypertensive men than in normotensive men. In addition, plasma 8-iso-PG F2 $\alpha$ levels were correlated with plasma hs-CRP levels. Furthermore, the present study clearly demonstrated that the order parameter (S) of RBCs was correlated with plasma 8-iso-PG F2 $\alpha$ and hs-CRP levels, indicating that reduced membrane fluidity of RBCs was 


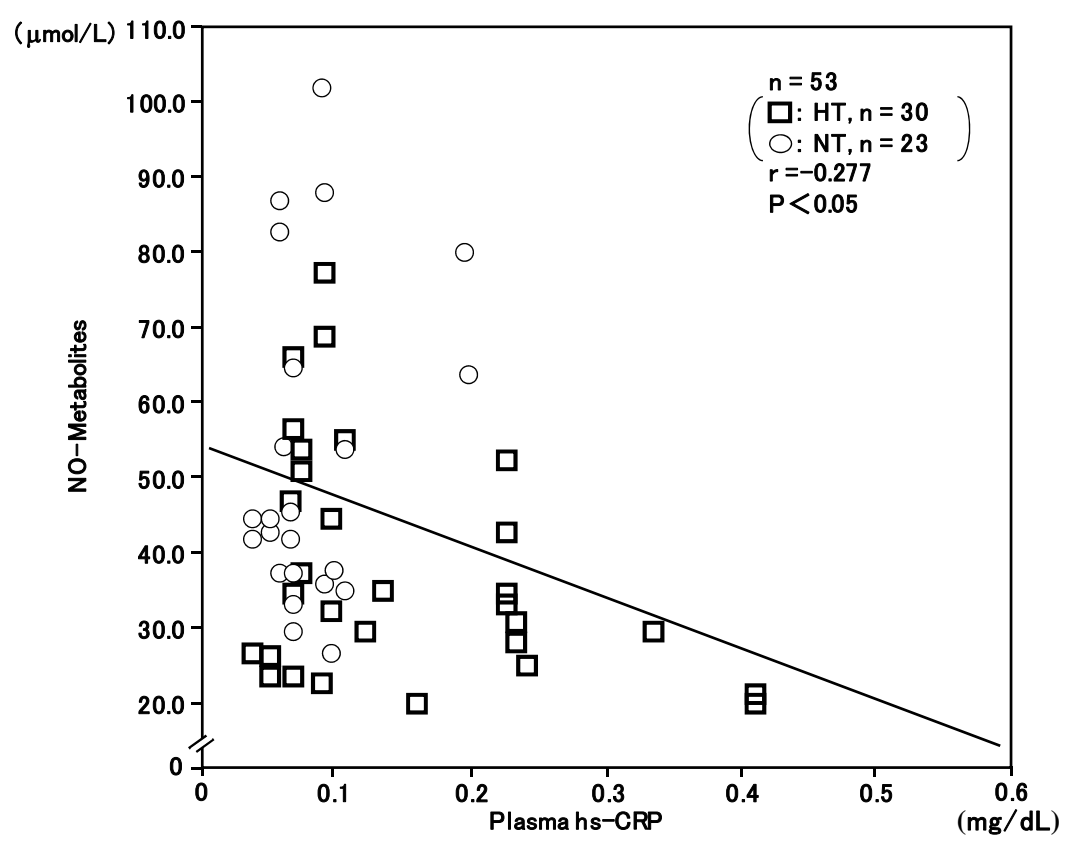

Figure 4. Inverse correlation between plasma high-sensitivity C-reactive protein (hs-CRP) and plasma NO-metabolite levels in hypertensive and normotensive men.

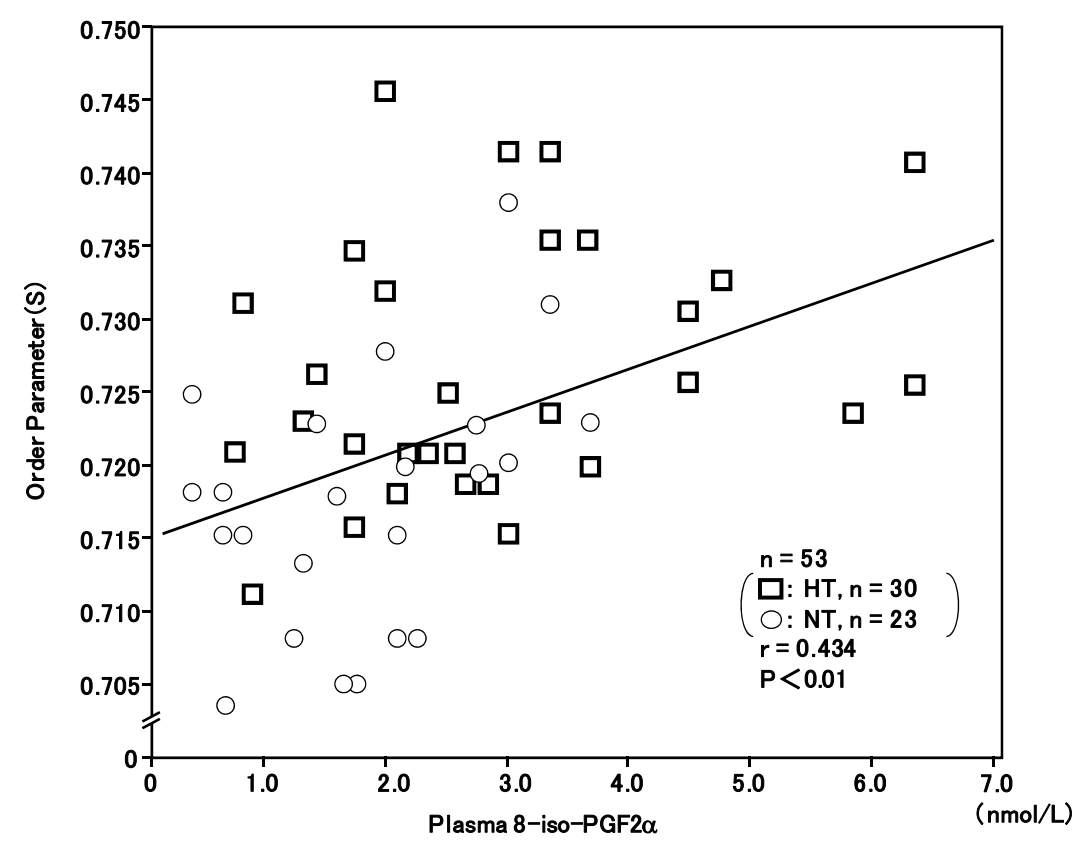

Figure 5. Correlation between plasma 8-iso-PG F2 $\alpha$ levels and membrane fluidity (order parameter; S) of red blood cells (RBCs) in hypertensive and normotensive men. The greater the value of the order parameter (S) was, the lower the membrane fluidity of RBCs was [17-20].

associated with increased oxidative stress and inflammation. Multivariate regression analysis also showed that, after adjustment for general risk factors, plasma 8-iso-PG F $2 \alpha$ and hs-CRP were insignificant determinants of membrane fluidity of RBCs, respectively. One hypothe- sis is that the reduction in membrane fluidity of RBCs with increased oxidative stress and inflammation could cause a disturbance in the blood rheologic behavior and the microcirculation in hypertension.

It has been already shown that shear rate, shear stress 


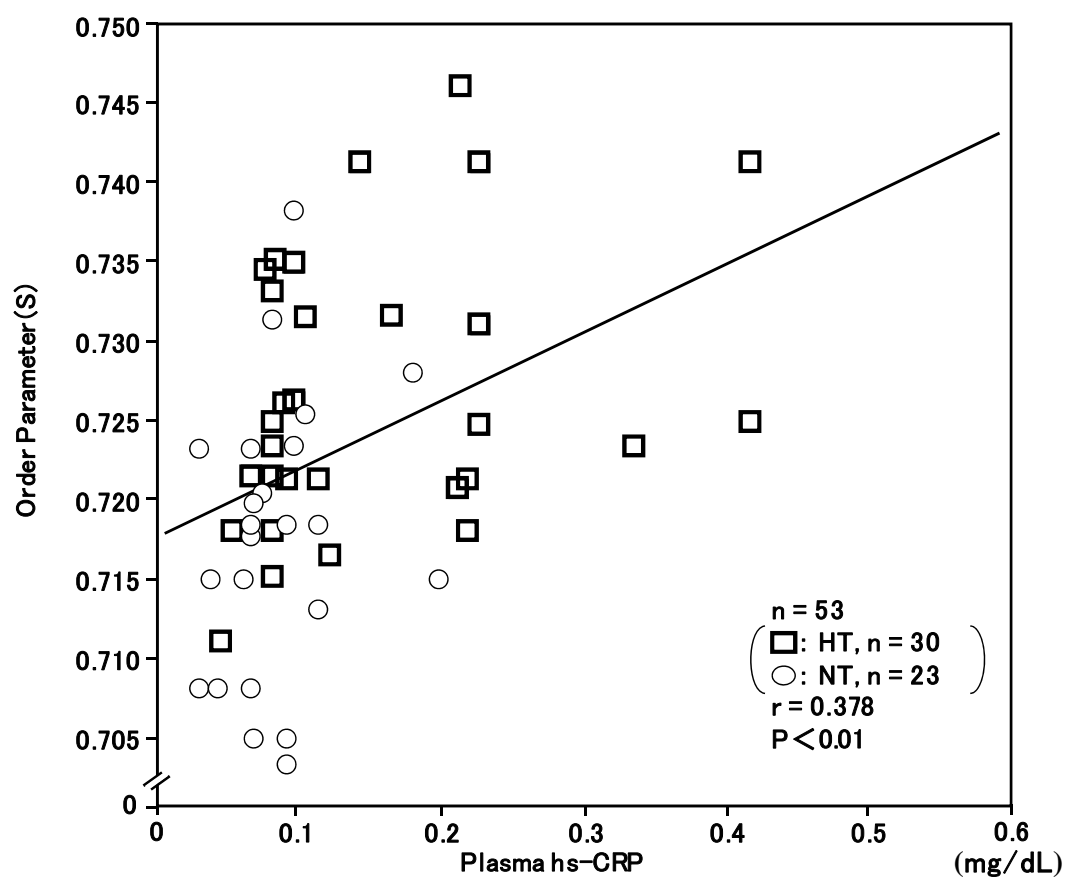

Figure 6. Correlation between plasma high-sensitivity C-reactive protein (hs-CRP) levels and membrane fluidity (order parameter; S) of red blood cells (RBCs) in hypertensive and normotensive men.

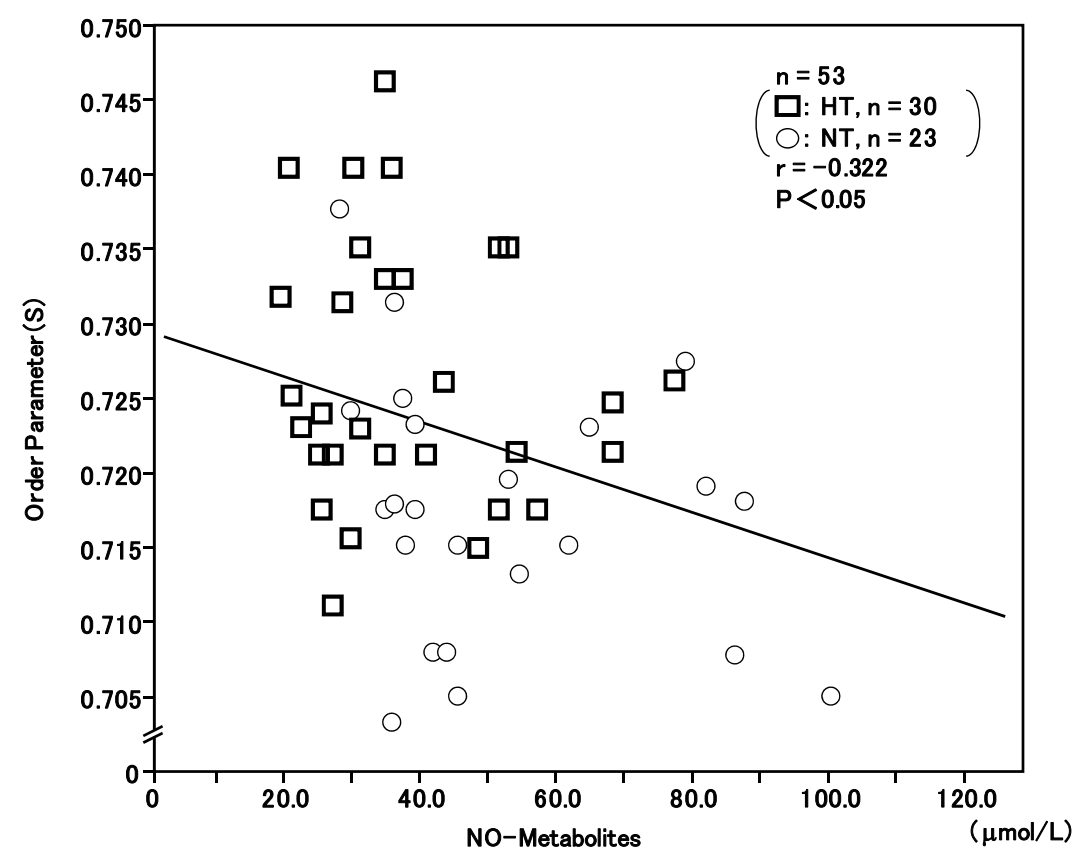

Figure 7. Inverse correlation between plasma NO-metabolite levels and membrane fluidity (order parameter; S) of red blood cells (RBCs) in hypertensive and normotensive men.

and blood viscosity were correlated with membrane fluidity of RBCs [23]. The finding proposed that in vivo shear forces might participate in the control of RBC membrane fluidity, and that RBCs might adapt their membrane properties to blood flow conditions. It was also demonstrated that RBC membranes might become more rigid after myocardial infarction, which could contribute to the decreased RBC deformability and the in- 
Table 2. Multivariate regression analysis for predicting order parameter (S) of RBCs.

\begin{tabular}{lccc}
\hline & SRC & t-value & P value \\
\hline Age (y.o.) & -0.292 & -1.745 & 0.0879 \\
Body mass index $\left(\mathrm{kg} / \mathrm{m}^{2}\right)$ & -0.268 & -1.763 & 0.0848 \\
Total cholesterol (mg/dL) & -0.266 & -1.691 & 0.0978 \\
Fasting plasma glucose (mg/dL) & 0.138 & 1.117 & 0.2698 \\
Systolic blood pressure (mm Hg) & 0.263 & 2.098 & 0.0415 \\
Plasma 8-iso-PGF2a (nmol/L) & 0.350 & 2.479 & 0.0170 \\
Plasma hs-CRP (mg/dL) & 0.274 & 2.087 & 0.0426 \\
\hline
\end{tabular}

$\mathrm{R}^{2}=0.404, \mathrm{n}=53, \mathrm{~F}=4.364, \mathrm{P}=0.0009$; SRC: standard regression coefficient; 8-iso-PGF2 $\alpha$ : 8-iso-prostaglandin F2 $\alpha$; hs-CRP: high-sensitivity Creactive protein.

creased blood viscosity in this group of patients [24]. Cazzola et al. [25] reported that the membrane fluidity of RBCs was decreased in the obese subjects, and proposed that a decrease in RBC membrane fluidity could contribute to a reduction of the rate of blood flow and the oxygen diffusion through the RBC membranes and its exchange with tissues. It might be possible that alterations in RBC membrane fluidity with increased oxidative stress and inflammation would be strongly linked to the progression of circulatory disorders.

The precise mechanisms by which oxidative stress and CRP could affect the membrane functions remain unclear. In the present study, we demonstrated that lower membrane fluidity of RBCs was associated with decreased plasma NO-metabolite levels. In an in vitro study presented earlier, we observed that an NO donor significantly improved membrane fluidity of RBCs in hypertensive subjects, suggesting that NO could have a beneficial effect on the rheologic behavior of RBCs and the microcirculation in hypertension [17]. Recently, it was shown that endothelium-dependent vasodilation was impaired in subjects with elevated oxidative stress levels [2, $26,27]$. On the other hand, it was also demonstrated that CRP by itself might directly impair the NO synthase expression in human aortic endothelial cells in vitro [12]. The present study showed that plasma 8-iso-PG F2 $\alpha$ and hs-CRP levels were inversely correlated with plasma NO-metabolite concentration in the overall analysis of hypertensive and normotensive men. It can be speculated that interactions between oxidative stress and inflammation might strongly impair membrane fluidity of RBCs, at least in part, by reducing NO-bioavailability, although direct actions of oxidative stress and CRP on membrane structural and functional properties cannot be fully excluded $[28,29]$. Further studies should be performed to assess more precisely the roles of oxidative stress and inflammation in the regulation of membrane functions in hypertension.

\section{CONCLUSION}

In summary, the results of the present study demonstrated that both plasma 8-iso-PG F2 $\alpha$ and hs-CRP levels were elevated in hypertensive men compared with normotensive men. Furthermore, it was shown that reduced membrane fluidity of RBCs was associated with increased plasma 8-iso-PG F2 $\alpha$ and hs-CRP levels, and decreased plasma NO metabolite levels, suggesting that abnormalities in RBC membranes in hypertension might be strongly related to increased oxidative stress and inflammation, and endothelial dysfunction. Although this is a cross-sectional and correlative study in Japanese men, the results of the present study could provide a hypothesis that associations of oxidative stress and inflammation might have a close correlation with impaired rheologic behavior of RBCs and microcirculatory dysfunction in hypertensive men.

\section{ACKNOWLEDGEMENTS}

This study was supported in part by grants-in-aid for scientific research from the Ministry of Education, Science, Sports, Culture and Technology of Japan (15590604, 18590658, 20590710, 23590901).

\section{REFERENCES}

[1] Heistad, D.D., Wakisaka, Y., Miller, J., Chu, Y. and PenaSilva, R. (2009) Novel aspects of oxidative stress in cardiovascular diseases. Circulation Journal, 73, 201-207. doi:10.1253/circj.CJ-08-1082

[2] Schulz, E., Gori, T. and Munzel, T. (2011) Oxidative stress and endothelial dysfunction in hypertension. $H y$ pertension Reseach, 34, 665-573. doi:10.1038/hr.2011.39

[3] Rahsepar, A.A., et al. (2011) Prooxidant-antioxidant balance and antioxidized LDL antibody level values and cardiac function in patients with coronary artery disease. Cardiology, 122, 203-209. doi:10.1159/000339273

[4] Whaley-Connell, A., McCullough, P.A. and Sowers, J.R. (2011) The oxidative stress in the metabolic syndrome. Review in Cardiovascular Medicine, 12, 21-29.

[5] Dhawan, V. and Jain, S. (2004) Effect of garlic supplementation on oxidized low density lipoprotein and lipid peroxidation in patients of essential hypertension. $\mathrm{Mo}$ lecular and Cellular Biochemistry, 266, 109-115. doi:10.1023/B:MCBI.0000049146.89059.53

[6] Rodrigo, R., et al. (2007) Relationship between oxidative stress and essential hypertension. Hypertension Research, 30, 1159-1167. doi:10.1291/hypres.30.1159

[7] Vassalle, C., Botto, N., Andreassi, M.G., Berti, S. and Biagini, A. (2003) Evidence for enhanced 8-isoprostane plasma levels, as index of oxidative stress in vivo, in patients with coronary artery disease. Coronary Artery Disease, 14, 213-218. doi:10.1097/01.mca.0000063504.13456.c3

[8] Wang, B., et al. (2006) Association of plasma 8-isoprostane levels with the presence and extent of coronary 
stenosis in patients with coronary artery disease. Atherosclerosis, 184, 425-430.

[9] Libby, P., Ridker, P.M. and Maseri, A. (2002) Inflammation and atherosclerosis. Circulation, 105, 1135-1143. doi:10.1161/hc0902.104353

[10] Lagrand, W.K., et al. (1999) C-reactive protein as a cardiovascular risk factor: More than an epiphenomenon? Circulation, 100, 96-102.

[11] Lloyd-Jones, D.M., Liu, K., Tian, L. and Greenland, P. (2006) Narrative review: Assessment of C-reactive protein in risk prediction for cardiovascular disease. Annals of Internal Medicine, 145, 35-42.

[12] Venugopal, S.K., Devaraj, S., Yuhanna, I., Shaul, P. and Jialal, I. (2002) Demonstration that C-reactive protein decreases eNOS expression and bioactivity in human aortic endothelial cells. Circulation, 106, 1439-1441. doi:10.1161/01.CIR.0000033116.22237.F9

[13] Qamirani, E., Ren, Y., Kuo, L. and Hein, T.W. (2005) C-reactive protein inhibits endothelium-dependent NOmediated dilatation in coronary arterioles by activating p38 kinase and NAD(P)H oxidase. Arteriosclerosis, Thrombosis and Vascular Biology, 25, 995-1001. doi:10.1161/01.ATV.0000159890.10526.1e

[14] Järvisalo, M.J., et al. (2002) Elevated serum C-reactive protein levels and early arterial changes in healthy children. Arteriosclerosis, Thrombosis and Vascular Biology, 22, 1323-1328. doi:10.1161/01.ATV.0000024222.06463.21

[15] Zicha, J., Kunes, J. and Devynck, M.A. (1999) Abnormalities of membrane function and lipid metabolism in hypertension. American Journal of Hypertension, 12, 315-331.

[16] Tsuda, K. and Nishio, I. (2003) Membrane fluidity and hypertension. American Journal of Hypertension, 16, 259-261. doi:10.1016/S0895-7061(02)03257-0

[17] Tsuda, K, Kimura, K., Nishio, I. and Masuyama, Y. (2000) Nitric oxide improves membrane fluidity of erythrocytes in essential hypertension: An electron paramagnetic resonance investigation. Biochemical and Biophysical Research Communications, 275, 946-954. doi:10.1006/bbrc.2000.3408

[18] Tsuda, K., Kinoshita, Y., Kimura, K., Nishio, I. and Masuyama, Y. (2001) Electron paramagnetic resonance investigation on modulatory effect of $17 \beta$-estradiol on membrane fluidity of erythrocytes in postmenopausal women. Arteriosclerosis, Thrombosis and Vascular Biology, 21, 1306-1312. doi:10.1161/hq0801.093507

[19] Tsuda, K., et al. (1987) Electron spin resonance studies of erythrocytes from spontaneously hypertensive rats and humans with essential hypertension. Hypertension, 9, III-19-III-24.

[20] Tsuda, K., Kinoshita-Shimamoto, Y., Kimura, K. and Nishio, I. (2003) Nitric oxide is a determinant of membrane fluidity of erythrocytes in postmenopausal women: An electron paramagnetic resonance investigation. American Journal of Hypertension, 16, 244-248. doi:10.1016/S0895-7061(02)03261-2

[21] Tsuda, K. (2010) Oxidative stress and membrane fluidity of red blood cells in hypertensive and normotensive men: An electron spin resonance investigation. International Heart Journal, 51, 121-124. doi:10.1536/ihj.51.121

[22] Tsuda, K. (2012) Associations between high-sensitivity C-reactive protein and membrane fluidity of red blood cells in hypertensive elderly men: An electron spin resonance study. International Journal of Hypertension, 2012, Article ID: 292803. doi:10.1155/2012/292803

[23] Le Sang Quan, K.H., Levenson, J., Del Pino, M., Simon, A. and Devynck, M.A. (1993) In vivo shear flow and erythrocyte membrane fluidity in hypertensive patients. British Journal of Clinical Pharmacology, 36, 437-443. doi:10.1111/j.1365-2125.1993.tb00392.x

[24] Saldanha, C., et al. (1999) Impairement of the erythrocyte membrane fluidity in survivors of acute myocardial infarction. A prospective study. Clinical Hemorheology and Microcirculation, 20, 111-116.

[25] Cazzola, R., Rondanelli, M., Russo-Volpe, S., Ferrari, E. and Cestaro, B. (2004) Decreased membrane fluidity and altered susceptibility to peroxidation and lipid composition in overweight and obese female erythrocytes. Journal of Lipid Research, 45, 1846-1851. doi:10.1194/jlr.M300509-JLR200

[26] Loffredo, L., et al. (2007) Oxidative-stress-mediated arterial dysfunction in patients with peripheral arterial disease. European Heart Journal, 28, 608-612. doi:10.1093/eurheartj/ehl533

[27] Annuk, M., Zilmer, M., Lind, L., Linde, T. and Fellström, B. (2001) Oxidative stress and endothelial function in chronic renal failure. Journal of American Society of $\mathrm{Ne}$ phrology, 12, 2747-2752.

[28] Zou, C.G., Agar, N.S. and Jones, G.L. (2001) Oxidative insult to human red blood cells induced by free radical initiator AAPH and its inhibition by commercial antioxidant mixture. Life Sciences, 69, 75-86. doi:10.1016/S0024-3205(01)01112-2

[29] Lenfant, F., et al. (2000) Lidocaine inhibits potassium efflux and hemolysis in erythrocytes during oxidative stress in vitro. General Pharmacology, 34, 193-199. doi:10.1016/S0306-3623(00)00060-4 